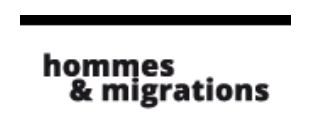

Hommes \& migrations

Revue française de référence sur les dynamiques

migratoires

$1288 \mid 2010$

Langues et migrations

\title{
Héritage et pratiques linguistiques des descendants d'immigrés en France
}

\section{Stéphanie Condon et Corinne Régnard}

\section{(apenEdition \\ Journals}

\section{Édition électronique}

URL : http://journals.openedition.org/hommesmigrations/854

DOI : 10.4000/hommesmigrations.854

ISSN : 2262-3353

\section{Éditeur}

Musée national de l'histoire de l'immigration

\section{Édition imprimée}

Date de publication : 1 novembre 2010

Pagination : 44-56

ISSN : 1142-852X

\section{Référence électronique}

Stéphanie Condon et Corinne Régnard, « Héritage et pratiques linguistiques des descendants d'immigrés en France », Hommes \& migrations [En ligne], 1288 | 2010, mis en ligne le 29 mai 2013, consulté le 01 mai 2019. URL : http://journals.openedition.org/hommesmigrations/854 ; DOI 10.4000/hommesmigrations.854 


\section{Héritage et pratiques linguistiques des descendants d'immigrés en France}

Par Stéphanie Condon, chercheuse à l'Institut national d'études démographiques (Ined) et Corinne Régnard, démographe

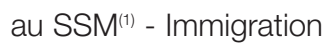

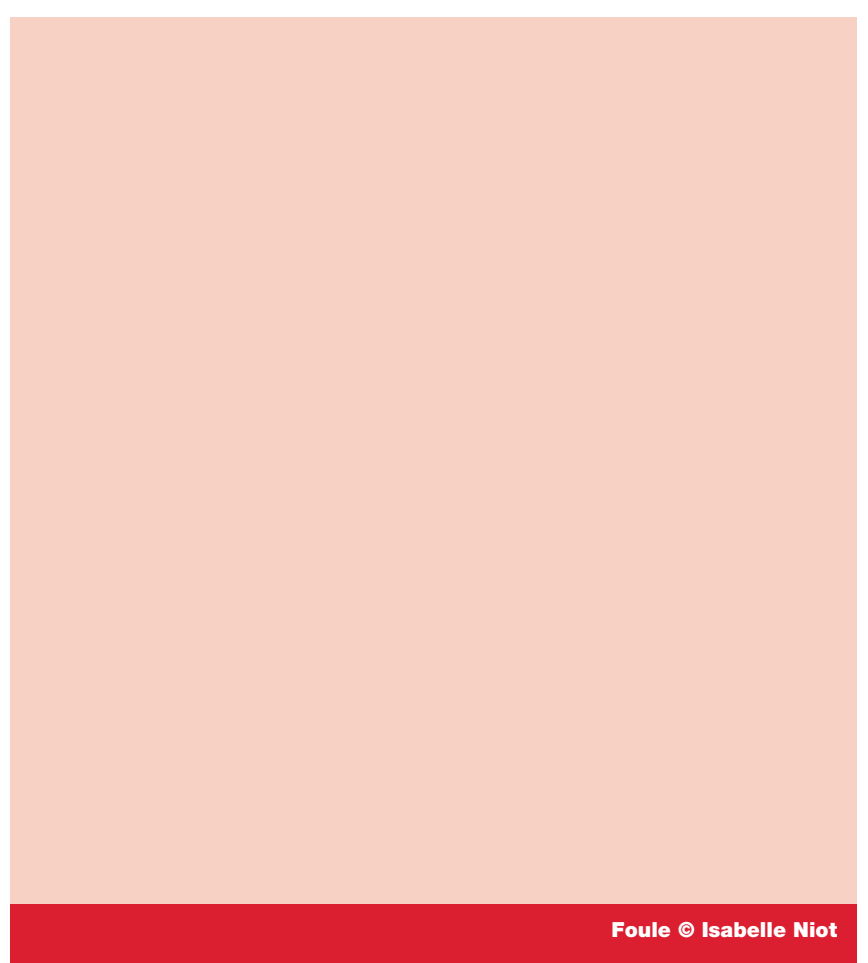

Loin d'être un obstacle à l'intégration, le plurilinguisme dans les familles ayant un lien avec la migration est à considérer comme une ressource potentielle. Une enquête menée par l'Ined et l'Insee

permet d'évaluer la transmission du français et des langues étrangères aux descendants d'immigrés par leurs parents. La pratique de différentes langues en famille et leur niveau de maîtrise résultent d'une négociation entre l'apprentissage de la langue majoritaire en France et la place accordée à l'héritage linguistique du pays d'origine. 
Les quinze dernières années témoignent d'une pérennisation des questions sur les pratiques linguistiques dans les enquêtes sociodémographiques. Le plus souvent, ce questionnement se situe dans une problématique d'intégration des populations immigrées. ${ }^{(2)}$ Mais, étant donné le rôle de la langue dans les transmissions culturelles entre générations et dans la construction de l'identité, la thématique de la transmission des langues en famille - qu'elles soient régionales ou étrangères - a été traitée de façon plus large dans des enquêtes en population générale en France ${ }^{(3)}$.

Pour ce qui concerne les immigrés, la maîtrise de la langue majoritaire du pays d'immigration constitue bien évidemment un facteur d'intégration professionnelle et sociale. Puis, pour leurs enfants nés et élevés dans ce pays, outre son importance dans la vie familiale, la transmission des langues des parents peut représenter une ressource mobilisable sur le marché du travail, une ouverture du champ des possibles dans le domaine professionnel, social, culturel.

La pratique bilingue ou plurilingue résulte de l'héritage d'une ou plusieurs langues étrangères en famille et de l'acquisition de la langue majoritaire du pays à l'école et dans d'autres sphères de la vie quotidienne. Dans ce contexte, les enfants d'immigrés apprennent à jouer un rôle de médiateur lorsque les parents ne maîtrisent pas suffisamment la langue de l'environnement pour gérer certaines activités, notamment celles concernant la scolarité. Plutôt que de signifier que ces jeunes vivent entre deux chaises ou cultures, leur héritage plurilingue combinerait une pratique du français les insérant dans leur génération, née et socialisée en France, et une pratique d'une ou deux langues étrangères qui les ancre dans leur famille élargie ${ }^{(4)}$.

En 1999, d'après l'enquête $\mathrm{EHF}^{(5)}, 14$ \% des jeunes accédant à l'âge adulte avaient été élevés dans une langue d'immigration et, dans la majorité des cas, ces langues avaient été transmises en même temps que le français. L'expérience de transmission plurilingue était alors devenue plus courante dans les générations les plus récentes.

\section{Analyser la transmission linguistique dans l'immigration}

Comme dans les autres enquêtes françaises précitées, l'enquête Trajectoires et Origines menée en 2008 par l'Ined et l'Insee (voir encadré ci-après) a tenu compte de la possibilité d'acquérir plusieurs langues en milieu familial, offrant aux répondants l'occasion de déclarer une langue autre que le français et de valoriser une pratique plurilingue. Nous nous proposons d'examiner ici l'héritage linguistique et le plurilinguisme des descendants d'immigrés ${ }^{(6)}$ vivant en France à partir des déclarations de ceux-ci sur des langues reçues de leur(s) parent(s) et la place du français 
et des langues étrangères dans le répertoire linguistique familial. Nous explorerons l'influence de quelques-uns des facteurs identifiés dans les études précédentes dans la transmission familiale des langues étrangères : la mixité du couple parental, l'âge à la migration des parents (à l'âge adulte ou pendant l'enfance), le maintien des liens au pays d'origine des parents.

Une question permettant une auto-évaluation du niveau de maîtrise des langues étrangères reçues par les parents ${ }^{(7)}$ éclaire, par ailleurs, sur le plurilinguisme des descendants à l'âge adulte. Cependant, ces derniers constituent un groupe très hétérogène, notamment en termes de l'histoire migratoire de leurs parents et de la connaissance du français avant cette migration. Pour tenir compte de la complexité des facteurs en jeu dans la transmission des langues étrangères ou de la pratique plurilingue $^{(8)}$, des études approfondies auprès de sous-populations sont nécessaires ${ }^{(9)}$. Toutefois, l'originalité de cette étude réside dans la prise en compte de l'héritage et des pratiques linguistiques des descendants.

\section{Plurilinguisme des descendants et place du français}

Par définition, les descendants d'immigrés sont nés en France et, sauf quelques rares exceptions, y ont tous été scolarisés, ce qui leur confère une bonne connaissance aussi bien à l'écrit qu'à l'oral de la langue française, cette dernière étant la langue des apprentissages scolaires et la langue véhiculaire des relations avec les pairs. Pour autant, issus d'au moins un parent immigré, leur enfance a pu baigner dans un plurilinguisme plus ou moins prégnant selon la composition du couple des parents (couple mixte, couple de deux parents immigrés originaires du même pays ou de deux pays différents), la volonté des parents de transmettre une ou plusieurs langues étrangères et la capacité de ceux-ci à participer à l'apprentissage de la langue française. En 2008, près d'un descendant d'immigrés sur deux (49\%, tableau 1) âgé de 18 à 50 ans et résidant en France métropolitaine déclare avoir reçu ${ }^{(10)}$ plusieurs langues pendant l'enfance de la part de ses parents. La plupart des plurilingues dans l'enfance sont en fait uniquement bilingues : très peu déclarent plus de deux langues durant l'enfance (5\%). Ce plurilinguisme de l'enfance est fortement influencé par la composition du couple de parents : il est très élevé lorsque les deux parents sont nés à l'étranger dans le même pays (67\%), moins élevé lorsqu'ils sont nés dans deux pays différents (47\%) et le fait d'avoir un parent né en France tend à le réduire sensiblement (26 \% de plurilingues lorsque la mère est née en France, 35 \% lorsque c'est le père qui y est né), faisant prévaloir une utilisation souvent exclusive du français. 


\section{Trajectoires et Origines (Te0), enquête sur la diversité des populations en France}

L'enquête TeO vise à décrire et analyser les conditions de vie et les trajectoires sociales des individus en fonction de leurs origines sociales et de leur lien à la migration vers la France métropolitaine.

Elle a été réalisée entre septembre 2008 et février 2009 par les enquêteurs de I'Insee auprès d'environ 22000 personnes nées entre 1948 et 1990, vivant dans un ménage ordinaire en France métropolitaine en 2008. Pour les descendants d'un immigré ou d'un parent né dans un DOM, le champ représentatif de l'enquête est limité aux individus nés après 1958.

Le questionnaire de $\mathrm{TeO}$ explore l'histoire migratoire, les parcours scolaires et professionnels, l'histoire résidentielle et les conditions de logement, la vie familiale, les modalités de transmission des langues et la religion. De façon transversale, il examine l'accès des individus aux biens et services (travail, logement, services, soins...) ainsi que les discriminations pouvant y faire obstacle.

L'enquête a été coordonnée conjointement par l'Institut national d'études démographiques (Ined) et l'Institut national de la statistique et des études économiques (Insee) avec la participation d'une équipe scientifique.

Elle a également bénéficié de l'appui financier de nombreux partenaires.

\section{Pour plus d'informations :}

Les tout premiers résultats ont été publiés en mars 2010 :

- Borrel C., Lhommeau B., "Être né en France d'un parent immigré", Insee Première, n¹287, mars 2010.

- Beauchemin C., Hamel C., Lesné M., Simon P. et l'équipe TeO,

"Discriminations : une question de minorités visibles", in Population et Sociétés, n 466, mars 2010.

Puis en octobre 2010, la synthèse des premiers résultats : Trajectoires et Origines. Enquête sur la diversité des populations en France. Premiers résultats, Documents de travail de l'Ined, Octobre 2010, 144 p. (bientôt en ligne sur le site de l'enquête).

- Le site de l'enquête : www.teo.site.ined.fr

Ce plurilinguisme s'exprime la plupart du temps par une pratique du français avec une langue étrangère. Seul un descendant sur dix $(9 \%)$ déclare qu'aucun de ses parents ne lui a parlé en français pendant son enfance : cela concerne très rarement les descendants ayant un parent né en France mais près d'un descendant sur cinq (19\%) dont les deux parents sont nés dans le même pays étranger. Pour ceux ayant des parents nés dans deux pays étrangers différents, il semblerait que le français s'impose le plus souvent comme langue de communication familiale (tableau 1). 


\section{Tableau 1. Transmission des langues et plurilinguisme pendant l'enfance des descendants diimmigrés selon le pays de naissance des parents}

\begin{tabular}{|c|c|c|c|c|c|}
\hline \multirow[b]{2}{*}{ FRÉQUENCE EN \% } & \multicolumn{2}{|c|}{$\begin{array}{l}\text { Couple de parents } \\
\text { "mixte (1)" }\end{array}$} & \multicolumn{2}{|c|}{$\begin{array}{l}\text { Couple de deux parents } \\
\text { nés à l'étranger }\end{array}$} & \multirow{2}{*}{$\begin{array}{l}\text { Ensemble } \\
\text { des } \\
\text { descendants } \\
\text { d'immigrés }^{(2)}\end{array}$} \\
\hline & $\begin{array}{l}\text { Père né } \\
\text { en France, } \\
\text { mère née } \\
\text { à l'étranger }\end{array}$ & $\begin{array}{l}\text { Père né à } \\
\text { l'étranger, } \\
\text { mère née } \\
\text { en France }\end{array}$ & $\begin{array}{l}\text { Parents } \\
\text { nés dans le } \\
\text { même pays } \\
\text { étranger }\end{array}$ & $\begin{array}{l}\text { Parents } \\
\text { nés dans } \\
\text { deux pays } \\
\text { étrangers } \\
\text { différents }\end{array}$ & \\
\hline \multicolumn{6}{|l|}{ MONOLINGUISME } \\
\hline $\begin{array}{l}\text { Uniquement } \\
\text { la langue française }\end{array}$ & 64,3 & 73,4 & 16,0 & 50,2 & 41,8 \\
\hline $\begin{array}{l}\text { Uniquement } \\
\text { une langue étrangère }\end{array}$ & 0,3 & 0,3 & 17,1 & 2,7 & 8,8 \\
\hline \multicolumn{6}{|l|}{ PLURILINGUISME } \\
\hline $\begin{array}{l}\text { Langue française } \\
\text { transmise par } \\
\text { les deux parents }\end{array}$ & 31,2 & 22,9 & 53,1 & 39,1 & 40,3 \\
\hline $\begin{array}{l}\text { Langue française } \\
\text { transmise par la mère }\end{array}$ & 0,7 & 3,0 & 5,0 & 4,5 & 3,8 \\
\hline $\begin{array}{l}\text { Langue française } \\
\text { transmise par le père }\end{array}$ & 2,8 & 0,2 & 7,4 & 2,0 & 4,3 \\
\hline $\begin{array}{l}\text { Uniquement plusieurs } \\
\text { langues étrangères }\end{array}$ & 0,7 & 0,1 & 1,5 & 1,6 & 1,0 \\
\hline Total & 100,0 & 100,0 & 100,0 & 100,0 & 100,0 \\
\hline $\begin{array}{l}\text { Nombre moyen } \\
\text { de langues parlées } \\
\text { pendant l'enfance }\end{array}$ & 1,4 & 1,3 & 1,7 & 1,6 & 1,5 \\
\hline $\begin{array}{l}\text { Fréquence } \\
\text { de plurilinguisme } \\
\text { pendant l'enfance }\end{array}$ & 35,4 & 26,2 & 67,0 & 47,2 & 49,4 \\
\hline $\begin{array}{l}\text { Fréquence } \\
\text { de l'absence totale } \\
\text { de la langue française } \\
\text { pendant l'enfance }\end{array}$ & 1,0 & 0,4 & 18,6 & 4,2 & 9,8 \\
\hline \multicolumn{6}{|c|}{$\begin{array}{l}\text { Source : Enquête Trajectoires et Origines, Ined-Insee, } 2008 . \\
\text { Champ : Descendants d'immigrés âgés de } 18-50 \text { ans résidant en France métropolitaine. } \\
\text { Exploitation : C. Régnard, S. Condon. } \\
\text { En italique : proportions calculées à partir d'effectifs observés inférieurs à } 50 .\end{array}$} \\
\hline \multicolumn{6}{|c|}{$\begin{array}{l}\text { Notes : } \\
\text { 1. Un parent né en France, l'autre né à l'étranger. } \\
\text { 2. Y compris les descendants dont on ne connaît pas le pays de naissance d'au moins un des deux parents. }\end{array}$} \\
\hline
\end{tabular}


Bien évidemment, la capacité à parler à ses enfants en français dépend de la connaissance de cette langue par les parents avant la migration (que nous ne connaissons pas à partir de cette enquête), sinon de la rapidité de l'acquisition de la langue depuis le début du séjour et d'une appropriation de la langue et des modes de pensée qui lui sont associés. De même, la transmission d'une langue suppose une pratique quotidienne, du moins en milieu familial, sinon dans les diverses sphères de la vie dans un pays où la langue est largement pratiquée. Pour ces raisons, l'âge des parents à la migration est une variable clef à prendre en compte dans les possibilités de transmission linguistique.

Les parents arrivés très jeunes auront pu suivre un enseignement en langue française au travers du système scolaire alors que pour ceux arrivés plus tard, n'ayant pas suivi de formation linguistique, le français sera surtout la langue de l'environnement social et professionnel, acquise peu à peu. Ainsi, lorsque les deux parents nés à l'étranger ont migré avant l'âge de 11 ans $^{(11)}$, ils ont transmis plus fréquemment le français que lorsqu'ils ont tous les deux migré après cet âge ( $97 \%$ contre $80 \%$, tableau 2). De même, cette transmission de la langue française est nettement plus souvent exclusive dans le cas d'une migration des deux parents à un jeune âge (60\% contre $15 \%$ ) et le plurilinguisme des descendants donc moins fréquent (37\% contre $68 \%$ ).

\section{Tableau 2. Transmission de la langue française lorsque les deux parents sont nés à l'étranger selon l'âge à la migration des parents}

\begin{tabular}{|c|c|c|c|c|c|}
\hline EN \% & $\begin{array}{c}\text { Les deux } \\
\text { parents } \\
\text { sont } \\
\text { arrivés } \\
\text { avant l'âge } \\
\text { de } 11 \text { ans }\end{array}$ & $\begin{array}{l}\text { Le père } \\
\text { est arrivé } \\
\text { avant l'âge } \\
\text { de } 11 \text { ans, } \\
\text { la mère } \\
\text { après l'âge } \\
\text { de } 11 \text { ans }\end{array}$ & $\begin{array}{l}\text { Le père } \\
\text { est arrivé } \\
\text { après l'âge } \\
\text { de } 11 \text { ans, } \\
\text { la mère } \\
\text { avant l'âge } \\
\text { de } 11 \text { ans }\end{array}$ & $\begin{array}{l}\text { Les deux } \\
\text { parents } \\
\text { sont } \\
\text { arrivés } \\
\text { après l'âge } \\
\text { de } 11 \text { ans }\end{array}$ & $\begin{array}{l}\text { Ensemble } \\
\text { des } \\
\text { descendants } \\
\text { d'immigrés }^{(1)}\end{array}$ \\
\hline $\begin{array}{l}\text { Fréquence } \\
\text { de plurilinguisme } \\
\text { pendant l'enfance }\end{array}$ & 36,8 & 57,1 & 60,0 & 67,5 & 64,6 \\
\hline $\begin{array}{l}\text { Fréquence } \\
\text { de la présence } \\
\text { de la langue française } \\
\text { pendant l'enfance }\end{array}$ & 97,4 & 94,7 & 94,6 & 80,4 & 83,1 \\
\hline $\begin{array}{l}\text { Uniquement la langue } \\
\text { française }\end{array}$ & 60,5 & 37,6 & 35,3 & 14,6 & 20,0 \\
\hline \multicolumn{6}{|c|}{$\begin{array}{l}\text { Source : Enquête Trajectoires et Origines, Ined-Insee, } 2008 . \\
\text { Champ : Descendants d'immigrés âgés de } 18-50 \text { ans résidant en France métropolitaine dont les deux parents sont } \\
\text { nés à l'étranger. Exploitation : C. Régnard, S. Condon. } \\
\text { En italique : proportions calculées à partir d'effectifs observés inférieurs à } 50 .\end{array}$} \\
\hline
\end{tabular}




\section{Principales langues autres que le français parlées pendant l'enfance avec les parents}

La diversité et la fréquence des langues ou dialectes étrangers cités par les descendants reflètent à la fois l'importance des flux migratoires passés et l'apparition de nouveaux flux. Deux informations complémentaires ont été recueillies dans l'enquête $\mathrm{TeO}$ : d'abord, la ou les langues parlées lors des échanges entre la mère ou le père et le descendant pendant son enfance et, ensuite, la désignation d'une langue "familiale", c'est-à-dire celle qui est déclarée comme la plus utilisée. Elle correspond donc à une appréciation de celle qui était prédominante dans les échanges lorsque l'enquêté se retrouvait en famille. Il ne faut d'ailleurs pas négliger la présence d'autres personnes que les parents pendant ces échanges (frères, sceurs, autres membres de la famille) où le français ou une langue étrangère pouvaient être utilisés, de façon alternée et selon le contexte. On pense, par exemple, au rôle des aînés de la fratrie dans la transmission du français au sein de la famille, à travers l'organisation des jeux ou la pratique narrative $e^{(12)}$.

Comme on pouvait s'y attendre, le maintien d'une langue étrangère dans le répertoire linguistique familial est le plus fréquent lorsque les deux parents sont nés à l'étranger. La moitié $(50 \%)$ des descendants de ces familles déclare que le français a été la langue la plus utilisée. Les autres langues étrangères déclarées sont l'arabe (20 \% de l'ensemble) ou le berbère $(3 \%)$, le portugais $(10 \%)$, les langues africaines du Niger-Congo $(5 \%)^{(13)}$, l'espagnol (4\%), l'italien (4\%) et le turc (2\%). Pour les descendants d'immigrés dont l'un des parents est né en France, d'autres langues apparaissent parfois comme langue la plus utilisée. Cette pratique est certainement liée à l'histoire familiale du parent né en France (par exemple, lui-même étant descendant d'immigré) sinon à une bonne connaissance d'une langue étrangère acquise (par exemple, celle du conjoint) par ce parent et donc un choix de transmission plurilingue.

Compte tenu de la très grande diversité des flux d'immigration, et donc des caractéristiques des descendants, nous avons choisi de nous focaliser sur quelquesunes des origines les plus importantes (graphique 1). Parmi les descendants de deux parents nés dans le même pays, il y a globalement peu de différences entre les pratiques des mères et des pères en termes de transmission d'une langue étrangère. Les descendants de deux parents nés en Algérie estiment que le français était le plus souvent la langue familiale, même s'il était utilisé moins souvent dans les échanges individuels avec la mère ou le père. Au contraire, les descendants de deux parents nés en Turquie ont le souvenir que le français était moins souvent la langue familiale que dans son utilisation avec l'un ou l'autre parent. Il en va de même pour les descendants de migrants nés au Portugal. 
Graphique 1 : Présence du français ou d'une autre langue parmi les langues reçues de la mère et du père et langue la plus souvent utilisée en famille selon le pays de naissance des parents 
En ce qui concerne les descendants de couples "mixtes" (où l'un des parents est né en France), comme nous l'avons dit auparavant, la langue familiale désignée est quasiment toujours le français. Toutefois, la transmission des langues étrangères perdure très souvent. Ainsi, les pères nés au Portugal semblent transmettre autant, voire légèrement plus, la langue du pays d'origine que les mères qui y sont nées. Alors que c'est un peu plus le fait des mères nées en Algérie, en Espagne ou en Italie que des pères. Se combinent des facteurs familiaux, culturels, identitaires ou liés au niveau de scolarisation ou de maitrise du français pour expliquer ces choix de transmission. Enfin, l'analyse révèle une transmission d'autres langues que le français par le parent né en France, souvent la langue étrangère transmise par son conjoint immigré ; un indicatif de l'histoire familiale du parent né en France et de la proximité communautaire dans la formation de certains couples. Ainsi, environ $3 \%$ des pères nés en France dont la conjointe est née au Portugal ont transmis le portugais à leur enfant enquêté, et près de $10 \%$ des descendants de mère née en Algérie déclarent que le père né en France leur a parlé en arabe ou en berbère.

\section{Maîtrise des langues étrangères apprises en famille}

Le plurilinguisme tel que nous l'avons approché ici correspond à celui "hérité" pendant l'enfance et ne présume donc en rien du plurilinguisme des descendants d'immigrés en 2008. Francophones de par leur vie en France et leur scolarisation dans le système français, nous l'avons vu, une large majorité des descendants d'immigrés ont grandi dans un contexte familial où une ou plusieurs langues étrangères étaient parlées. Or, si certains à l'âge adulte restent bilingues seulement au niveau d'une compréhension plus ou moins bonne de la langue étrangère reçue, d'autres ont acquis une très bonne maîtrise de cette langue.

Près de la moitié ( $42 \%$ ) des descendants n'ont déclaré que la langue française comme langue héritée des parents. Parmi ceux qui déclarent avoir reçu une langue étrangère, $72 \%$ ont deux parents nés dans le même pays. Nous nous limiterons donc ici à l'examen du niveau de maîtrise par les descendants de ce dernier groupe des langues étrangères reçues pendant l'enfance, et ceci pour quelques origines.

Il s'avère que plus de la moitié des descendants ayant deux parents nés dans le même pays étranger déclarent avoir une très bonne maittrise de la principale ${ }^{(14)}$ langue étrangère apprise dans ces familles (bien comprise et facilement parlée, voire parlée, lue, écrite). Les descendants de parents nés au Portugal, en Espagne ou en Turquie sont nombreux à lire et à écrire respectivement le portugais, l'espagnol ou le turc. 
Sauf pour les descendants de parents nés au Viêtnam, rares sont ceux qui déclarent ne comprendre que quelques mots de la langue étrangère transmise par les parents (tableau 3). Les groupes où les personnes ayant une très bonne maîtrise, notamment en lecture et en écriture, sont les plus souvent concernés par les cours de langue (la moitié des descendants de parents nés au Portugal ou en Turquie, 41 \% de ceux dont les parents sont nés au Maroc). Par exemple, 64 \% des descendants de deux parents nés au Portugal déclarant pouvoir lire et écrire le portugais ont suivi des cours, par rapport à $27 \%$ de ceux déclarant seulement bien le comprendre et le parler. Ce sont aussi dans ces groupes qu'on trouve les visites les plus fréquentes au pays d'origine des parents avant l'âge de 18 ans, moments où ces langues pouvaient être pratiquées avec des personnes au-delà de la famille en France. Notons que, même pour les descendants de parents nés au Viêtnam, le fait d'avoir visité le pays des parents au moins une fois concerne une large majorité des enquêtés et que près des trois quarts de ces visites se sont faites avant l'âge de 18 ans.

\section{Le maintien du lien avec le pays d'origine des parents}

Nous avons regardé aussi le rôle de la langue dans l'utilisation de médias ${ }^{(15)}$ du pays des parents. Par exemple, pour les descendants de parents nés en Turquie, 91 \% de ceux qui maîtrisent le turc à l'écrit utilisent les médias de ce pays contre 50 \% de ceux qui le parlent bien sans l'écrire. Pour les descendants de parents nés au Portugal, les proportions sont respectivement $63 \%$ et 44 \%. La maîtrise de la langue et l'utilisation des médias sont certainement liées : un fort intérêt pour les affaires du pays d'origine du ou des parents, de la vie politique au succès des équipes sportives, motive les descendants à améliorer leur niveau de compétences dans la langue. Sans compter que l'utilisation des nouvelles technologies joue également un rôle dans cette amélioration.

Ainsi, la transmission linguistique, les séjours réguliers au pays d'origine des parents, le maintien de liens avec l'entourage "là-bas", le désir de se tenir au courant des affaires du pays s'imbriquent pour que ces descendants puissent bénéficier des atouts d'un espace de vie transnational. Cependant pour certains groupes, mis à part un projet migratoire des parents peut-être moins axé sur le retour au pays, les descendants n'ont pas pu bénéficier de séjours au pays des parents aussi fréquents, ni d'accès aussi facile aux cours dans les langues reçues par les parents ${ }^{(16)}$.

Un autre indicateur du maintien des langues étrangères comme langues familiales est le taux de retransmission par les descendants d'immigrés ${ }^{(17)}$. Malgré la prédominance $\mathrm{du}$ français dans les langues transmises aux descendants, une certaine proportion transmet à leur tour, à leurs propres enfants, les langues étrangères reçues des parents. 


\section{Tableau 3. Maîtrise de la langue étrangère héritée et liens au pays d'origine des parents (en \%)}

\begin{tabular}{|c|c|c|c|c|c|c|c|c|}
\hline \multirow{2}{*}{$\begin{array}{l}\text { Pays de } \\
\text { naissance } \\
\text { des deux } \\
\text { parents }\end{array}$} & \multirow[b]{2}{*}{$\begin{array}{l}\text { Langue } \\
\text { étrangère } \\
\text { de } \\
\text { référence }\end{array}$} & \multirow[b]{2}{*}{$\begin{array}{l}\text { Cours } \\
\text { suivis } \\
\text { dans cette } \\
\text { langue }\end{array}$} & \multicolumn{4}{|c|}{ Langue étrangère de référence } & \multirow[b]{2}{*}{$\begin{array}{c}\text { Visites } \\
\text { au pays de } \\
\text { naissance } \\
\text { des } \\
\text { parents }\end{array}$} & \multirow{2}{*}{$\begin{array}{c}\text { Utilisation } \\
\text { des } \\
\text { médias du } \\
\text { pays de } \\
\text { naissance } \\
\text { des } \\
\text { parents }\end{array}$} \\
\hline & & & $\begin{array}{l}\text { Parlée, } \\
\text { lue, écrite }\end{array}$ & $\begin{array}{c}\text { Bien } \\
\text { comprise, } \\
\text { facilement } \\
\text { parlée }\end{array}$ & $\begin{array}{c}\text { Bien } \\
\text { comprise, } \\
\text { difficile- } \\
\text { ment } \\
\text { parlée }\end{array}$ & $\begin{array}{l}\text { Quelques } \\
\text { mots } \\
\text { compris }\end{array}$ & & \\
\hline Portugal & portugais & 49,6 & 59,8 & 23,3 & 15,0 & 1,9 & 99,5 & 54,5 \\
\hline Algérie & arabe & 27,4 & 14,9 & 58,5 & 25,1 & 6,4 & 90,1 & 49,5 \\
\hline Algérie & berbère & 2,6 & 4,5 & 52,8 & 33,7 & 9,0 & 90,2 & 46,5 \\
\hline Maroc & arabe & 40,7 & 23,1 & 60,6 & 14,1 & 2,1 & 97,3 & 60,4 \\
\hline Turquie & turc & 53,6 & 80,7 & 15,6 & 3,6 & 0,1 & 98,5 & 83,7 \\
\hline Sénégal & LDNC $^{(18)}$ & 4,0 & 19,3 & 46,2 & 28,0 & 6,4 & 86,9 & 65,6 \\
\hline Viêtnam & vietnamien & 8,9 & 9,0 & 48,3 & 22,6 & 20,0 & 61,3 & 27,4 \\
\hline Espagne & espagnol & 44,4 & 68,0 & 24,5 & 7,6 & 0,0 & 97,9 & 48,5 \\
\hline
\end{tabular}

\section{Le plurilinguisme, une ressource sous-évaluée?}

Les enquêtes statistiques permettent de recenser les langues transmises, d'évaluer l'héritage plurilingue dans les familles ayant un lien avec la migration, et, par l'étude du niveau de maîtrise des langues étrangères reçues, de cerner le potentiel d'une ressource.

Les répondants à l'enquête $\mathrm{TeO}$ semblent avoir saisi l'occasion ${ }^{(19)}$ de citer les langues utilisées en famille, éléments forts dans la transmission culturelle et de leur identité. Est-ce que ces déclarations traduisent une reconnaissance de la ressource que représente le plurilinguisme? Et est-ce que les parents transmetteurs ont fait le choix de maintenir les langues de leur enfance? Ce ne sont pas des questions auxquelles nous pouvons répondre ici. Mais on peut dire que ce n'est certainement pas le cas pour toutes les langues car, dans des contextes d'immigration, les différentes langues en présence sont souvent hiérarchisées (et c'est le cas de la France ${ }^{(20)}$ ), selon le prestige social de certaines langues apportées par l'immigration et la dévalorisation d'autres. Certains dialectes ou langues pratiqués dans les pays anciennement sous administration française ont souvent été dévalorisés par rapport au français dans un "marché linguistique ${ }^{(21) " ~ q u i ~ a ~ p r o m u ~ l e ~ f r a n c ̧ a i s ~ c o m m e ~ l a n g u e ~ d e ~ l a ~ r e ́ u s s i t e ~ s o c i a l e . ~}$ 
Ce qui est frappant dans les résultats de l'étude est la richesse des langues déclarées. Dans les familles où les deux parents sont originaires du même pays d'origine, l'utilisation de la langue de l'environnement, le français, va le plus souvent de pair avec celle de la ou des langues d'enfance des parents, en alternance ou selon le contexte. Lorsque l'un des parents est né en France, l'utilisation du français prévaut mais, on l'a vu, la langue étrangère parlée par le parent immigré est souvent transmise, pratique parfois renforcée par l'utilisation de cette langue également par le parent né en France.

\section{Définitions et cadrage statistique}

Selon la définition adoptée par le Haut Conseil à l'Intégration, un "immigré" est une personne née étrangère à l'étranger et résidant en France.

Un descendant direct d'immigré (ou, dans le texte, "descendant d'immigré") est une personne née en France métropolitaine d'au moins un parent né étranger à l'étranger.

En 2008, les descendants âgés de 18 à 50 ans sont un peu plus de 3 millions et représentent 11,5\% de l'ensemble des 18-50 ans résidents en France métropolitaine à cette date-là. Ils sont légèrement plus jeunes que l'ensemble des résidents, âgés en moyenne de 31,7 ans (contre 34,6 ans).

L'origine de l'ascendance est dite "mixte", quand un descendant direct d'immigré n'a qu'un seul de ses parents qui est immigré. Dans ce cas, son autre parent est non immigré, soit né français en France ou à l'étranger, soit né étranger en France.

Plus de deux descendants sur cinq (43 \%) ont des parents en couple "mixte" et dans la majorité des cas $(61 \%)$ le père est français et la mère d'origine étrangère. Lorsque les descendants sont issus d'un couple de deux parents immigrés, leurs deux parents ont la même origine dans près de neuf cas sur dix (88 \%).

Nous entendrons par "origine des descendants d'immigrés", le pays de naissance du parent immigré lorsque le descendant est issu d'un couple "mixte", du père lorsque les deux parents sont immigrés.

Près de deux descendants sur cinq sont originaires d'Europe du Sud (Espagne, Italie, Portugal - $39 \%$ ) et $35 \%$ le sont d'un des trois pays du Maghreb (20\% d'Algérie). Le quart restant est surtout composé de descendants originaires d'Afrique subsaharienne, du Sud-Est asiatique (Cambodge, Laos, Viêtnam) et des autres pays de I'Union européenne à 27.

Enfin, certains descendants ont été élevés par un seul parent ou d'autres proches. On parlera donc du "père ou d'une figure paternelle" et de la "mère ou d'une figure maternelle". 


\section{Enfin, les questions sur le niveau de maîtrise des langues étrangères reçues révèlent que plus de la moitié des descendants a un très bon niveau à l'oral, mais aussi à l'écrit. On cerne donc un potentiel, certainement sous-estimé, voire ignoré par la société comme par les descendants eux-mêmes ${ }^{(22)}$.}

\section{Notes}

1. Service statistique ministériel.

2. En France, l'enquête MGIS (Mobilité géographique et insertion sociale, 1992) menée par Ined-Insee sous la responsabilité de Michèle Tribalat. Sur les pratiques linguistiques, à partir de cette enquête, voir l'article de Patrick Simon, "L'acculturation linguistique : utilisation du français et transmission de la langue des immigrés à leurs enfants" in Migrants-Formation, n 108, 1997, pp. 53-66.

3. Étude de l'histoire familiale (EHF, Insee, 1999); Histoire de vie et construction des identités (HdV, INSEE-INED, 2002)

4. Christine Deprez, Les enfants bilingues : langues et familles, Paris, Didier-CREDIF, 1994.

5. François Héran, "Une approche quantitative de l'intégration linguistique en France", in Hommes et Migrations $\mathrm{n}^{\circ} 1252,2004$, pp. 10-24.

6. Le terme qui sera utilisé dans cet article pour se référer aux personnes nées en France ayant au moins un parent (ou figure parentale) immigré, comme détaillé dans l'encadré en fin d'article.

7. Langue majoritaire du pays ou dialecte régional du pays d'origine des parents immigrés.

8. Par exemple, les choix parentaux de transmission, le rôle des différents membres de la famille, les contextes d'utilisation des différentes langues.

9. Voir par exemple Christine Deprez, op. cit.; Alexandra Filhon, "Langues d'ici et d'ailleurs : transmettre l'arabe et le berbère en France", in Les cahiers de l'Ined, n 163, Paris, Institut national d'études démographiques, 2009.

Ou encore les travaux publiés dans la revue électronique Glottopol (www.univ-rouen.fr).

10. La question a été formulée ainsi : Quelles sont la ou les langues que vous parlait votre mère / votre père quand vous étiez enfant?

11. Le seuil d'âge est variable selon les analyses. Ici l'âge a été fixé à 11 ans, âge qui correspond à l'entrée au collège, incluant donc les migrants ayant fait tout ou partie de leur scolarité élémentaire en France. À noter que l'âge à la migration des parents, lorsque les deux parents sont nés à l'étranger, n'est connu que dans 86 \% des cas.

12. Fabienne Leconte, "Les langues africaines en France", in Cahiers de l'Observatoire des pratiques linguistiques, n², Paris, Éditions Didier, 2008, pp. 57-63.

13. Près d'une centaine de langues de cette famille ont été citées, les plus répandues étant le bambara, le comorien, le lingala, le malinké, le mandingue, le peul, le sango, le senoufo, le sérère, le soninké, le soussou ou encore le wolof.

14. Pour $81 \%$ des descendants dont les deux parents sont nés en Algérie, la principale langue étrangère utilisée en famille a été l'arabe et pour 19 \%, c'est le berbère. On l'appelle ici la "langue de référence".

15. La question rassemble divers types de médias : radio, télévision, presse, Internet. Leur utilisation suppose des niveaux de compétence différents, mais nous ne pouvons pas aller plus loin dans l'analyse. Rappelons que certains médias, notamment des pays anciennement sous administration française, sont francophones.

16. Danièle Moore, Plurilinguismes et école, Paris, Les Éditions Didier, 2006 ; et l'article de Salem Chaker sur l'enseignement du berbère en France, dans Hommes et Migrations, n ${ }^{\circ}$ 1252, 2004, pp. 25-33.

17. Ceci est étudié ailleurs par F. Héran, op.cit. et par A. Filhon, op.cit. Les analyses à partir de TeO seront publiées dans l'ouvrage collectif à venir.

18. Langue ou dialecte de la famille des langues nigéro-congolaises ; voir note 13.

19. Avec toutes les réserves en matière de sous ou surestimation des déclarations.

20. Rapport de Jacques Leclerc, item "France" dans L'Aménagement linguistique dans le monde, Québec, TLFQ, Université Laval, http://www.tlfq.ulaval.ca/axl

21. Pierre Bourdieu, Ce que parler veut dire : Léconomie des échanges linguistiques, Paris, Fayard, 1991.

22. Cyril Trimaille, "Pratiques langagières chez les adolescents d'origine maghrébine" in H. et M., $\mathrm{n}^{\circ} 1252,2004$, pp. 66-73. 